\title{
Degradation Control for Electric Vehicle Machines using Nonlinear Model Predictive Control
}

\author{
L. Samaranayake Senior Member IEEE, S. Longo Senior Member IEEE
}

\begin{abstract}
Electric machines (motors and generators) are over actuated systems. In this paper we show how to exploit this actuation redundancy in order to mitigate machine degradation while simultaneously ensuring that the desired closed loop performance is maintained. We formulate a multi-objective optimization problem with a cost function having terms representing closed loop performance and component degradation for an inverter-fed permanent magnet synchronous motor. Such machines are important as they are widely used as the prime mover of commercial electric vehicles. The resulting optimal control problem is implemented online via a Nonlinear Model Predictive Control (NMPC) scheme. The control framework is validated for standard vehicle drive cycles. Results show that the NMPC scheme allows for better closed loop performance and lower degradation than standard industrial controllers such as the field oriented control method. Hence, this work demonstrates how the remaining useful life of a machine can be increased by appropriate controller design without compromising performance.
\end{abstract}

Index Terms-Degradation, Permanent Magnet Synchronous Motor, Nonlinear Model Predictive Control, Optimal Control.

\section{INTRODUCTION}

\section{A. Motivation}

C ONVENTIONALLY, the electric machine operating as the prime mover in electric and hybrid electric vehicles has been considered as one of the most reliable and most rugged components in the entire electric powertrain. It is expected to operate over 20,000 hours or 15 years, whichever comes first, without degrading in terms of its power delivering capability and efficiency. This happens because of the use of high quality raw materials (e.g. rare earth materials in permanent magnets, high purity copper in windings, etc.) and conservative designs, as shown in [1], [2], [3], [4] and [5]. Due to the same reasons, however, it is one of the most expensive components in an electric vehicle powertrain, which negatively affects their popularity. Therefore, in electric machine design for electric vehicles, there exists a compromise between their rate of degradation and their costs [6], [7].

Degradation associated with winding insulations [8] [9], magnets [10] [11] [12], and bearings [13] [14] have been addressed in isolation. However, in electric vehicles applications, the machine is a main subsystem in the powertrain and therefore such individual considerations are not sufficient. In addition, unlike the industrial motors which are usually subjected to known static load demands, these machines have

L. Samaranayake is with the Department of Electrical and Electronic Engineering, Faculty of Engineering, University of Peradeniya, 20400 Peradeniya, Sri Lanka (e-mail:lilantha@ee.pdn.ac.lk). S. Longo is with the Advanced Vehicle Engineering Centre, Cranfield University, Bedfordshire, United Kingdom, MK43 0AL (e-mail:s.longo@cranfield.ac.uk). Corresponding author: S. Longo. to cater for dynamic torque and speed demands. It is therefore of high importance to have a degradation-aware control technique that will allow for satisfactory demand tracking performance similar to or better than conventional controllers. One approach to compensate for such design trade-offs is to introduce optimal methods to machine control. So far this approach has been ignored in the literature, possibly due to the practicalities of implementing computational expensive algorithms for the power electronics. Techniques such as receding horizon control are becoming increasingly popular due to the improvements in computational power and algorithm performance [15], and we can now assume that, with careful design, such techniques can be implemented in real-time [16][18]. For example Direct MPC [19] or Finite Control Set MPC [20], [21], [22] combine control and modulation blocks inside a single MPC controller, which enables very fast dynamics and better steady state control in power electronic applications.

\section{B. State of the art degradation control of electric machines}

We define degradation as the accumulated time integral of losses of the machine over its operational life time (more details are provided in the sequel). Simple degradation-aware control algorithms, based on loss minimization, exist and these have been implemented within the conventional dq-current controller frameworks [23] [25]. Irrespective of whether such techniques are implemented by analytically solving the loss minimizing equations [26], by using polynomials to approximate it [27], or by using fuzzy logic [28], their generic structure can be captured by the one depicted in Fig. 1. In all such approaches, the inner current controller in the conventional Field Oriented Control (FOC) [24] receives the $\mathrm{d}$-axis and q-axis current references $\left(i_{d}^{r e f}, i_{q}^{\text {ref }}\right)$ from the loss minimizing algorithm, to which the torque reference $\left(\tau^{r e f}\right)$, or the speed reference $\left(\omega^{r e f}\right)$, will be the input. However, no significant degradation minimization improvement has been reported in the latter against conventional control techniques such as $i_{d}=0$ or Maximum Torque Per Ampere (MTPA) control [29].

One fundamental reason for the insignificant gain is due to the fact that loss minimization algorithms are only used to determine $i_{d}^{r e f}$ and $i_{q}^{r e f}$. Beyond that point it is often a simple Proportional Integral (PI) current controller for the dqcurrent loops that generates the dq-voltage references $v_{d}^{r e f}$ and $v_{q}^{r e f}$. The main drawback of this approach is that, if the PI controllers are tuned to respond faster, then the overshoots will be high. On the other hand, if they are tuned to reduce the overshoot, then the response will be sluggish [30], [31], [32]. Unfortunately, both high overshoots and sluggish responses increase losses [33]. Hence, to minimize losses effectively and 


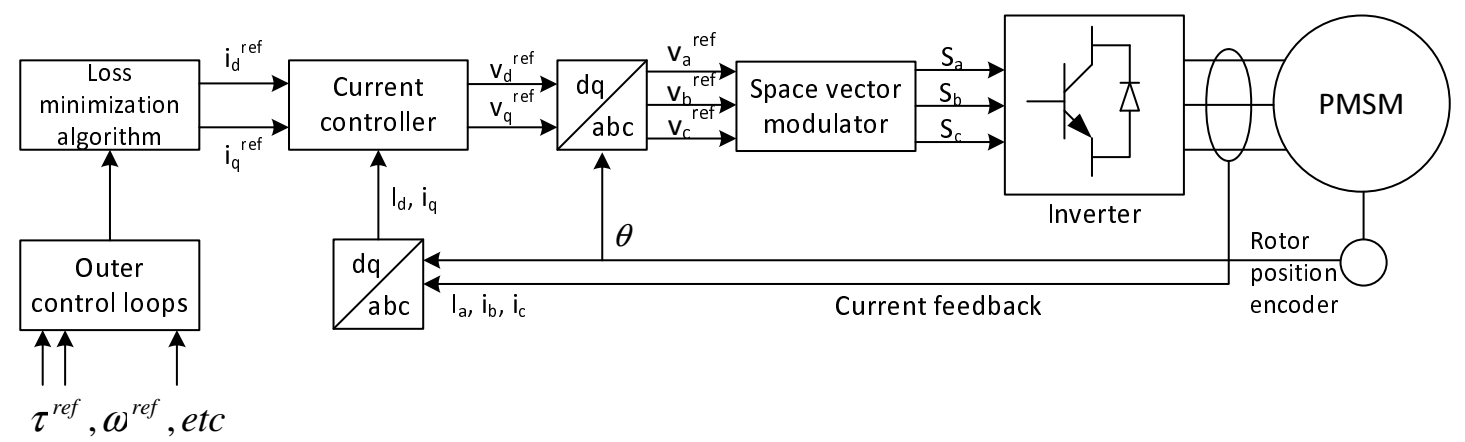

Fig. 1: Generic field oriented control structure of a Permanent Magnet Synchronous Motors (PMSM) accommodating loss minimization.

consequently the degradation, the control technique should ideally give a fast response without a large overshoot [33], [34].

Nonlinear Model Predictive Control (NMPC) [15], at the cost of a more complex algorithm, offers the flexibility to define cost functions with opposing requirements simultaneously. A model of the plant is necessary in order to derive the optimal control law that may be subjected to constraints. Only the first control move of the prediction horizon is usually applied at each sample. This process is repeated in a receding horizon fashion, taking reference input changes and output disturbances into account, as per the standard Model Predictive Control (MPC) scheme [35].

\section{Proposed control framework}

Application of MPC in electric machine control has less than a decade of history. In general, the current and rotor position information is used as output feedback. Control signals $\left(v_{d}^{r e f}, v_{q}^{r e f}\right.$ in Fig. 2) are derived, subjected to the constraints. Unlike previous approaches where the cost function consists of only the current errors $\left(i_{d}^{r e f}-i_{d}, i_{q}^{r e f}-i_{q}\right)$ or the speed error $\left(\omega^{r e f}-\omega\right)$, here we use a blend of degradation and torque error to form a multi-objective cost, which enables the controller to simultaneously deliver good transient performance, while minimizing degradation of the machine. The non-convex multiobjective cost function, together with the nonlinear constraints and the machine model are needed to set up the NMPC problem. The basic block diagram of the NMPC scheme, where a Permanent Magnet Synchronous Motor (PMSM) is used as the electric machine, is shown in the Fig. 2.

The main insight that justifies the need for optimal control for electric machines is the fact that the degradation rate can be improved without affecting the closed loop referencetracking performance. This is possible thanks to the inherent redundancies in the control system, that will be explained next. The electromagnetic torque $\tau_{e}$ of the PMSM is a function of both $i_{d}$ and $i_{q}$, where the unconstrained torque contours in the $i_{d}-i_{q}$ plane typically exist as shown in Fig. 3 .

Accordingly, there exists more than one combination of $\left\{i_{d}, i_{q}\right\}$ to generate the required $\tau_{e}$, which makes the PMSM an over actuated system. One of the contributions of this work is that we show how to exploit this redundancy to mitigate degradation, while simultaneously providing the desired torque-tracking performance. This is achieved by the following:

1) The energy-loss-based electric machine degradation cost function (which was introduced by the authors in [33]) is combined with the machine performance cost function to form a multi-objective cost, which is then used in the NMPC set up.

2) A conventional second-order PMSM model is used for the dynamic constraints of the NMPC. A novel higherorder PMSM model is derived and used as a plant, in order to achieve simulation results closer to reality.

3) Results are validated via a case study. Here, two standard drive cycles for urban and urban-motorway mixed driving have been used to investigate improvements in the form of remaining useful life of the PMSM in an electric vehicle.

\section{Related work}

NMPC has already been proposed for hybrid and electric vehicles applications. For example, it is used to find the optimal power split between the internal combustion engine and the electric machine of a hybrid electric vehicle in [36] to minimize fuel costs. Real-time NMPC is used in [16] for the control of a laboratory tower crane to minimize the tracking error. The implementation uses a fast NMPC, which will not necessarily return the optimal solution. However, NMPC is shown to be implemented in real-time. In [17], NMPC with a quadratic cost function is also implemented in real-time using state of the art hardware on an autonomous tractor-trailer reversing problem. Very high degree of steering accuracy is reported in this study. Furthermore, a constrained optimal control problem is solved in real-time to minimize the torque error using linearized PMSM models, with experimental results, in [18]. Linear MPC has been used experimentally for fault tolerant speed control of induction machines in [37]. Other theoretical derivations related to on-board power management in electric vehicles using MPC can be found in [38], [39].

\section{E. Organization of the paper}

The paper is organized as follows: Section II presents the machine model, degradation model and the degradation 


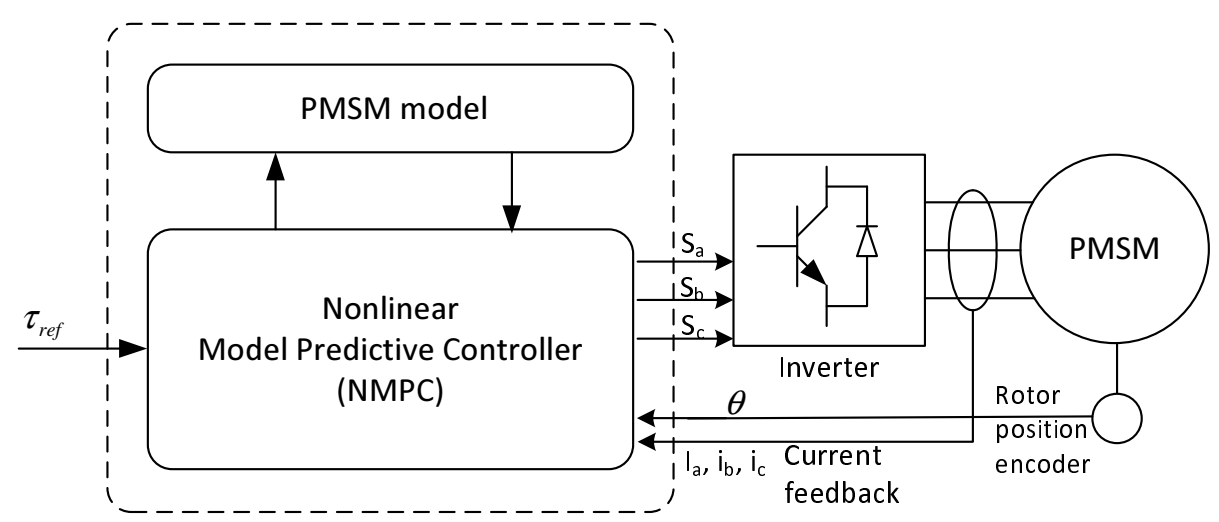

Fig. 2: Structure of the NMPC for PMSMs.

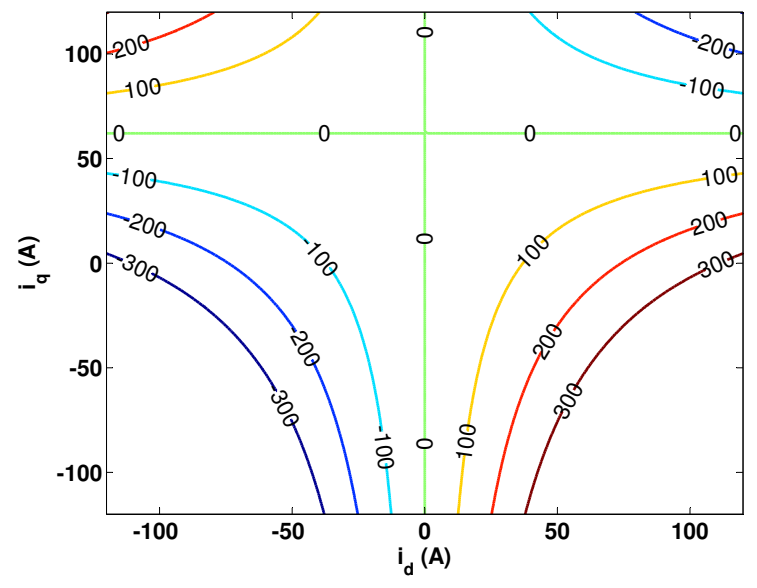

Fig. 3: Typical torque (in Nm) contours of the PMSM without voltage and current constraints.

metrics used to setup the optimal control problem. The NMPC design is presented in Section III. Simulation experiments followed by analysis and discussion of the results are presented in Section IV. A case study of applying the control scheme on a commercial electric vehicle machine degradation problem is presented in Section V. The paper is concluded with proposed future work in Section VI.

\section{MODELING OF MACHINE DYNAMICS AND DEGRADATION}

In this section, we present the conventional nonlinear model of the PMSM. We also derive the degradation model using machine losses and obtain the metrics to quantify the degradation experimentally. In this section, the time argument $(t)$ is omitted from time-dependent variables deliberately to improve readability. In addition, a novel higher-order model of the PMSM is also derived and presented in the Appendix. This higher-order model will be used in the experiments.

\section{A. Conventional machine model}

A PMSM, which is popular in the automotive industry for electric vehicle applications due to its high torque density and

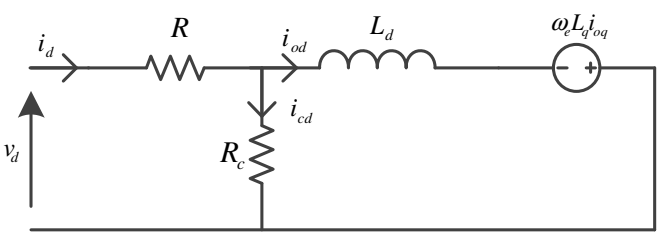

(a)

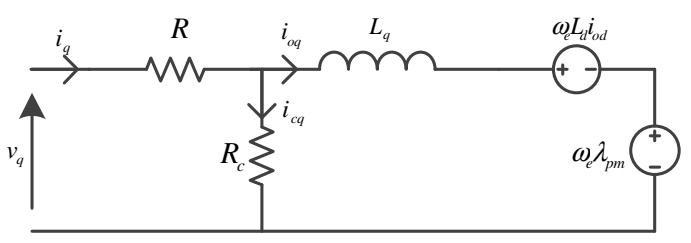

(b)

Fig. 4: Equivalent circuit of the conventional machine model.

minimum rotor losses, is modeled here. In order to avoid modeling the inverter and as a matter of supporting direct implementation, the dq-reference frame is used in deriving the model. The PMSM represented in the dq-reference frame consists of two circuits, one for the d-axis and another for the q-axis respectively, as opposed to the three circuits in the abcreference frame. The d-axis and q-axis equivalent circuits of the conventional PMSM model are shown in Fig. 4. Applying Kirchhoff's voltage and current laws in the d-axis circuit shown in Fig. 4 (a) and (b) results in

$$
\begin{aligned}
v_{d} & =L_{d} \frac{d i_{o d}}{d t}+R i_{d}-\omega_{e} L_{q} i_{o q} \\
i_{d} & =i_{o d}+i_{c d} \\
i_{c d} & =\frac{1}{R_{c}}\left(L_{d} \frac{d i_{o d}}{d t}-\omega_{e} L_{q} i_{o q}\right) .
\end{aligned}
$$

The q-axis circuit results in

$$
\begin{aligned}
v_{q} & =L_{q} \frac{d i_{o q}}{d t}+R i_{q}+\omega_{e} L_{d} i_{o d}+\omega_{e} \lambda_{p m} \\
i_{q} & =i_{o q}+i_{c q}
\end{aligned}
$$




$$
i_{c q}=\frac{1}{R_{c}}\left(L_{q} \frac{d i_{o q}}{d t}+\omega_{e} L_{d} i_{o d}+\omega_{e} \lambda_{p m}\right) .
$$

The PMSM considered in the work is an interior permanent magnet type with inverted saliency, i.e. $L_{d}<L_{q}$, which enables higher torque in the field weakening region [2]. The mechanical dynamics of the PMSM is governed by

$$
\frac{d \omega_{m}}{d t}=\frac{1}{J_{m}}\left(\tau_{e}-B_{m} \omega_{m}-\tau_{m}\right),
$$

where $\tau_{m}$ is the total load torque on the shaft, $\tau_{e}$ is the electromagnetic torque of the machine and $\omega_{m}=\frac{1}{n_{P}} \omega_{e}$ is the rotor shaft speed, where $n_{P}$ is the number of pole pairs of the PMSM. $J_{m}$ and $B_{m}$ are the equivalent inertia and the viscous friction of the rotor, respectively. However, the main focus here is on electrical losses contributing to the degradation. Hence, the slow mechanical dynamics have not been considered and the two voltages $v_{d}$ and $v_{q}$ become the inputs, which are equivalent to $v_{d}^{r e f}$ and $v_{q}^{r e f}$ in the dq-reference frame as shown in Fig. 2. The two currents $i_{d}$ and $i_{q}$ become the two outputs, which are equivalent to the dq-transformations of $i_{a}, i_{b}$ and $i_{c}$ in the three phase abc-reference frame (Fig. 2). By defining the input $u_{c}$, state $x_{c}$ and output $y_{c}$ vectors as

$$
\begin{aligned}
& u_{c}=\left[v_{d}, v_{q}\right]^{T}=\left[u_{1}, u_{2}\right]^{T} \\
& x_{c}=\left[i_{o d}, i_{o q}\right]^{T}=\left[x_{c 1}, x_{c 2}\right]^{T}=y_{c}
\end{aligned}
$$

the system can be expressed in state space format as

$$
\begin{aligned}
\dot{x_{c}} & =f_{c}\left(x_{c}, u_{c}\right) \\
f_{c} & =\left[f_{c 1}, f_{c 2}\right]^{T},
\end{aligned}
$$

where

$$
\begin{aligned}
f_{c 1} & =\frac{-R}{L_{d}\left(1+\frac{R}{R_{c}}\right)} x_{c 1}+\omega_{e} \frac{L_{q}}{L_{d}} x_{c 2} \\
& +\frac{1}{L_{d}\left(1+\frac{R}{R_{c}}\right)} u_{1}
\end{aligned}
$$

and

$$
\begin{aligned}
f_{c 2} & =-\omega_{e} \frac{L_{d}}{L_{q}} x_{1}+\frac{-R}{L_{q}\left(1+\frac{R}{R_{c}}\right)} x_{2} \\
& +\frac{1}{L_{q}\left(1+\frac{R}{R_{c}}\right)} u_{2}-\frac{\omega_{e} \lambda_{p m}}{L_{q}} .
\end{aligned}
$$

\section{B. Degradation models}

Our assumption here is that degradation is entirely related to losses [40]. The assumption holds in the sense that, if energy is not employed to do useful work, it will contribute to losses, degrading the components of the machine. The PMSM has several sources of power losses. Mainly they are: (a) stator copper losses, (b) stator iron core losses, (c) friction and windage losses and (d) mechanical losses. Magnet losses also exist, but, since their magnitude is comparatively small, they can be safely neglected. The relationship between the friction and windage losses and the mechanical losses are usually identified experimentally. Such studies reveal that the magnitudes of those losses contribute to only about $1-2 \%$ of the total losses [3]. Therefore they have not been taken into account in this study either.

The copper losses take place in all three windings and the total is their algebraic sum, which can be modeled using the well-known peak value currents in dq-reference frame as

$$
P_{C u}=\frac{3 R}{2}\left(i_{d}^{2}+i_{q}^{2}\right) \text {. }
$$

The iron losses occur in the stator iron laminations and they are modeled using the core loss resistance per phase $R_{c}$. Accordingly, the stator iron losses can be expressed by

$$
P_{F e}=\frac{3 R_{c}}{2}\left(i_{c d}^{2}+i_{c q}^{2}\right) \text {. }
$$

\section{Degradation metric}

The electric machines are designed for some rated speed, torque and power $\left(P_{\text {rated }}\right)$. In addition, depending on the design, manufacturing procedure, quality of the raw materials used and the manufacturing technology, the manufacturer recommends a useful lifetime $\left(t_{\text {life }}\right)$. For an electric machine with rated efficiency $\eta$, the total energy losses during its entire life time, if operated continuously, is

$$
W_{\text {losses }}=\int_{0}^{t_{\text {life }}}\left(\frac{1}{\eta}-1\right) P_{\text {rated }} d t .
$$

In reality, it will be operated intermittently. Let $0 \leq i \leq N$ be the index for operation cycles, where $N$ is the last operation completed. The energy losses during the $i^{\text {th }}$ continuous op-

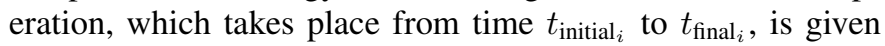
by

$$
W_{\text {losses }_{i}}=\int_{t_{\text {nnitial }_{i}}}^{t_{\text {final }_{i}}} P_{\text {input }_{i}}-P_{\text {output }_{i}} d t .
$$

The instantaneous output power is given in terms of the instantaneous shaft torque $\tau_{\text {output }_{i}}(t)$ and the shaft speed $\omega_{\text {output }_{i}}(t)$ as

$$
P_{\text {output }_{i}}=\tau_{\text {output }_{i}} \omega_{\text {output }_{i}} .
$$

Similarly, the corresponding instantaneous input power is given in terms of the instantaneous voltage of the inverter $V_{\text {inverter }_{i}}$ and the current $I_{\text {inverter }_{i}}$ as

$$
P_{\text {input }_{i}}=V_{\text {inverter }_{i}} I_{\text {}_{\text {nverter }}} .
$$

Depending on how the machine is operated, maintained and also depending on the environmental conditions and the age, the losses will vary. Therefore the cumulative machine losses will have to be taken into account in deriving the degradation metric. The Cumulative Loss Ratio (CLR) has been formulated by authors in [33]. In the CLR, the accumulated energy losses at the completion of $N$ cycles is compared with $W_{\text {losses }}$. Accordingly, the CLR is defined as

$$
\mathrm{CLR}=\frac{\sum_{i=1}^{N} W_{\text {losses }_{i}}}{W_{\text {losses }}} .
$$

It is interesting to note here that the CLR represents the fraction of the energy losses that the machine has been designed to tolerate, meaning that if this value is exceeded there will be a performance penalty. Therefore,

$$
\mathrm{RUL}=1-\mathrm{CLR}
$$


represents the Remaining Useful Life (RUL) of the machine. Consequently, the lower the CLR, the higher the RUL. As seen in (18), the CLR can be lowered by maintaining its numerator at a minimum. This suggests that the degradation can be minimized by minimizing the losses in the machine.

\section{NMPC DESIGN AND IMPLEMENTATION}

The NMPC algorithm computes a sequence of optimal control voltage inputs for the electric machine, which result in an optimal closed loop current profile in the sense that desired performance and minimum degradation is achieved over a finite horizon. After applying the first control voltage input of the sequence to the PMSM, the calculations are repeated at the next time step with the new current measurements, taking the reference torque trajectory $\left(\tau^{r e f}\right)$ also into account [35].

\section{A. Multi-objective cost function}

The cost function consists of two components: the degradation cost function $J_{D E G}$ and the closed loop performance cost function $J_{C L}$.

1) Degradation cost function: In order to minimize the degradation, as shown using the CLR, the energy losses must be minimized. Hence, the degradation cost function $J_{D E G}$ is defined as

$$
J_{D E G}=\int_{t_{i}}^{t_{f}}\left(P_{C u}+P_{F e}\right) d t .
$$

2) Closed loop performance cost function: The desired output of the PMSM is the electromagnetic torque given by

$$
\tau_{e}=\frac{3 n_{P}}{2}\left(\lambda_{p m}+i_{o d}\left(L_{m d}-L_{m q}\right)\right) i_{o q} .
$$

Hence, for a $\tau^{r e f}$ reference torque profile, the closed loop performance cost function can be defined as

$$
J_{C L}=\int_{t_{i}}^{t_{f}}\left(\tau^{r e f}-\tau_{e}\right)^{2} d t .
$$

Accordingly, when $J_{C L}$ is minimized, the absolute torque error $\tau^{r e f}-\tau_{e}$ will be minimized. This will ensure that the torque output of the machine follows the desired torque trajectory.

3) Combined cost functions: The multi-objective cost function $J$ can be formulated as

$$
J=\alpha J_{C L}+(1-\alpha) J_{D E G}
$$

where $0 \leq \alpha \leq 1$ is a tuning parameter to weight the contribution of each cost function. The advantage of this approach is that it gives the flexibility for the user to choose the desired operating point on a Pareto optimal frontier, as shown in Fig. 5, by selecting an arbitrary $\alpha$ between 0 and 1 . As $\alpha$ approaches 0 , more emphasis is given to the degradation minimization and less to the closed loop performance and vice versa. Hence, a given $\alpha$ will determine the best achievable degradation performance for a given closed loop performance or the best achievable closed loop performance for a given degradations performance, as depicted in Fig. 5. The parameter $\alpha$ can be treated as a tuning parameter that can chosen via trial and error, as it is often done in practice for multi-objective optimization. The user could start with $\alpha=1$, so that only

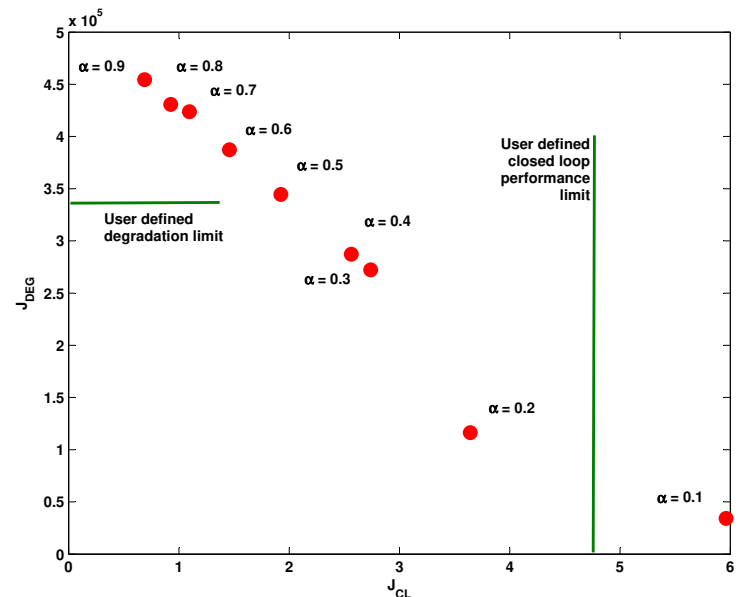

Fig. 5: Pareto optimal frontier for the multi-objective optimization problem together with an example of user defined maximum values for $J_{D E G}$ and $J_{C L}$.

performance is optimized and then decrease it and stop just before the performance reaches a level that is not acceptable any more. This process could be automated in a real setup with a simple search method which is not very time consuming.

\section{B. Nonlinear constraints}

Physical constraints arise from both the machine and the inverter, which powers the machine from some electrochemical energy storage. Usually, the power electronics from the system design is undersized, to protect the machine in case of an undetected fault. Further, safety factors are used to limit the maximum current for the protection of the inverter. Hence, the inverter current and the inverter voltage limitations need to be considered in the control design. The currents are such that the maximum of either $i_{d}$ or $i_{q}$ is $I_{\max }$. Similarly, the maximum voltage is $v_{d}$ or $v_{q}$ is $V_{\text {max }}$. In addition, $i_{d}$ will only be used for the purpose of field weakening. Therefore, the constraints can mathematically be formulated as

$$
\begin{aligned}
0 & \leq \sqrt{i_{d}^{2}+i_{q}^{2}} \leq I_{\max } \\
0 & \leq \sqrt{v_{d}^{2}+v_{q}^{2}} \leq V_{\max } \\
-I_{\max } & \leq i_{d} \leq 0 .
\end{aligned}
$$

The existence of such physical nonlinear constraints further justifies the use of a nonlinear constrained control approach.

\section{Optimal controller problem}

The optimal control problem is defined as

$$
\min _{u_{c}(\cdot)} J\left(x_{c}(\cdot), u_{c}(\cdot), t_{i}, t_{f}\right)
$$

subject to

$$
\dot{x_{c}}(t)=f_{c}\left(x_{c}(t), u_{c}(t)\right)
$$

and

$$
x_{c L} \leq x_{c}(t) \leq x_{c U}, u_{L} \leq u_{c}(t) \leq u_{U}, \forall t \in\left[t_{i}, t_{f}\right],
$$


for given $t_{i}, t_{f}$ and initial state $x_{c}\left(t_{i}\right)$. The predicted states can be calculated by

$$
x(t)=x\left(t_{i}\right)+\int_{t_{i}}^{t_{f}} f_{c}\left(x_{c}(t), u_{c}(t)\right) d t, \forall t \in\left[t_{i}, t_{f}\right],
$$

where $t_{f}-t_{i}$ is the prediction horizon, $J\left(x_{c}(\cdot), u_{c}(\cdot), t_{i}, t_{f}\right)$ is the multi-objective cost function given in (23) and $f_{c}\left(x_{c}(t), u_{c}(t)\right)$ is the lower-order PMSM model derived in Section II B. In addition, $x_{c}(t)=\left[i_{o d}, i_{o q}\right]^{T}$ and $u_{c}(t)=$ $\left[v_{d}, v_{q}\right]^{T}$ are the states and the outputs of the controller, respectively. The constraints $x_{c L} \leq x_{c}(t) \leq x_{c U}$ and $u_{L} \leq$ $u_{c}(t) \leq u_{U}$ are as given in Section III B.

The inputs to the controller are the torque demand $\tau^{\text {ref }}$ and current feedback $i_{d}, i_{q}$. Since the speed control loop is avoided in the model, $\omega_{e}$ is also an input to the model, which is equivalent to $n_{p} \frac{d \theta}{d t}$, where $\theta$ is the rotor position as shown in Fig. 2. The controller outputs are the respective voltages in the d-axis and the q-axis, i.e. $v_{d}$ and $v_{q}$, which correspond to $v_{d}^{r e f}$ and $v_{q}^{r e f}$ as was shown in Fig. 2. It is assumed that $v_{d}$ and $v_{q}$ reach $v_{d}^{r e f}$ and $v_{q}^{r e f}$ with no delay. This is a practically valid assumption used in many power electronic applications [45].

\section{Simulation EXPERIMENTS AND ANALYSiS}

For the simulation studies, a Matlab ${ }^{\text {TM }}$ based tool, ICLOCS [42], is used to solve the optimal control problem. ICLOCS uses the freely available IPOPT solver [43].

The machine used in this study has a peak torque of 280 $\mathrm{Nm}$ and continuous torque of $140 \mathrm{Nm}$ at $3000 \mathrm{rpm}$ base speed and at $1000 \mathrm{~V}$ dc link voltage. The other machine parameters and the inverter parameters are given in TABLE I.

TABLE I: Machine and inverter electrical parameters

\begin{tabular}{cc}
\hline Parameter & Value \\
\hline Stator resistance $R$ & $0.26 \Omega$ \\
Core resistance $R_{c}$ & $33.74 \Omega$ \\
Number of pole pairs $n_{p}$ & 10 \\
Permanent magnet flux linkage $\lambda_{p m}$ & $0.18 \mathrm{Vs}$ \\
d-axis leakage inductance $L_{l d}$ & $1 \mathrm{mH}$ \\
q-axis leakage inductance $L_{l q}$ & $1 \mathrm{mH}$ \\
d-axis magnetizing inductance $L_{m d}$ & $2 \mathrm{mH}$ \\
q-axis leakage inductance $L_{m q}$ & $4.9 \mathrm{mH}$ \\
d-axis lumped inductance $L_{d}$ & $3 \mathrm{mH}$ \\
q-axis lumped inductance $L_{q}$ & $5.9 \mathrm{mH}$ \\
Maximum inverter voltage $V_{\max }$ & $1000 \mathrm{~V}$ \\
Maximum inverter current $I_{\max }$ & $120 \mathrm{~A}$ \\
\hline
\end{tabular}

The prediction horizon in the NMPC is $20 \mathrm{~ms}$ (40 samples), which is sufficient for the machine to reach steady state following a step input. The sampling time used in all the controller implementations is $t_{s}=0.5 \mathrm{~ms}$. This has been selected to be 10 times faster than the fastest time constant. On a CPU (Intel(R) Core ${ }^{\mathrm{TM}} \mathrm{i} 7-4600 \mathrm{U}$ at $2.1 \mathrm{GHz}$ ), the distribution of time IPOPT takes to solve the NMPC shows that on average it takes $15.4 \mathrm{~ms}$, while it can reach $90 \mathrm{~ms}$ when the torque reference undergoes large changes. Clearly, this would prevent real-time implementation. However, we are only interested here in providing a framework for an optimal solution to the degradation problem for benchmarking purposes.
We believe that a real-time solution could be achieved with bespoke solvers, optimized code, approximated control laws or careful hardware implementation (e.g. fixed-point arithmetics, parallelization and/or custom hardware) which is outside the scope of this work. There is an analogy of the work proposed here with previous work where computational resources are co-optimized within the MPC framework [47], [48]. There exist platforms such as 'FORCESPro' [49] to implement such co-optimized designs.

For comparison, our proposed controller is benchmarked against conventional controllers. These are: (a) $i_{d}=0$ control, which is the simplest method, and (b) Maximum Torque Per Ampere (MTPA) control, which is the standard industrial control design used for minimizing losses. These two techniques are briefly discussed in the next section.

\section{A. Review of conventional control methods}

In $i_{d}=0$ control, the d-axis reference current is always maintained at zero, while the q-axis reference current is obtained from the machine electromagnetic torque as $i_{q}^{r e f}=$ $\left(\frac{2}{3 n_{p} \lambda_{p m}}\right) \tau^{r e f}$.

The phasor diagram of the currents and voltages of the machine neglecting the resistances shown in Fig. 6, will be used to outline the MTPA control. According to the phasor

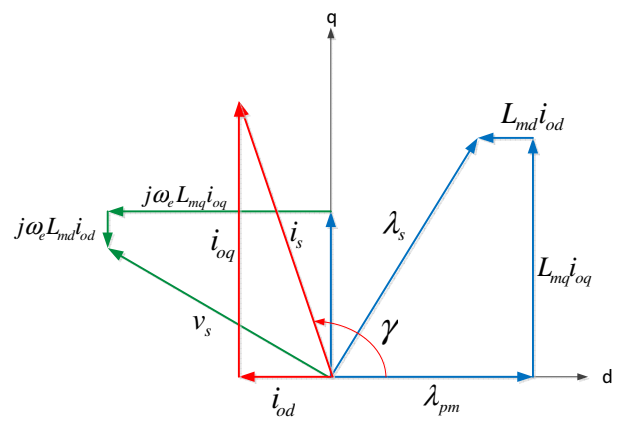

Fig. 6: Phasor diagram of the inverse salient PMSM.

diagram

$$
\begin{aligned}
& i_{o d}=i_{s} \cos (\gamma) \\
& i_{o q}=i_{s} \sin (\gamma)
\end{aligned}
$$

Hence, the electromagnetic torque per ampere can be obtained by substituting for $i_{o d}$ and $i_{o q}$ in the torque equation as

$$
\frac{\tau_{e}}{i_{s}}=\frac{3}{2} P\left[\lambda_{p m} \sin (\gamma)+\frac{1}{2}\left(L_{m d}-L_{m q}\right) i_{s} \sin (2 \gamma)\right] \text {. }
$$

By differentiating $\frac{\tau_{e}}{i_{s}}$ with respect to $\gamma$, it can be found that the current angle corresponding to the maximum torque per ampere $\gamma_{\max }$ can be given by

$$
\cos \left(\gamma_{\max }\right)=\frac{-\lambda_{p m} \pm \sqrt{\lambda_{p m}^{2}+8 i_{s}^{2}\left(L_{m d}-L_{m q}\right)^{2}}}{4 i_{s}\left(L_{m d}-L_{m q}\right)} .
$$

The corresponding $\mathrm{d}$-axis current can be found by $i_{s} \cos \left(\gamma_{\max }\right)$. Substituting for $\cos \left(\gamma_{\max }\right)$ and taking $i_{s}^{2}=$ $i_{o d}^{2}+i_{o q}^{2}$, the relationship between $i_{o d}$ and $i_{o q}$ for MTPA can 
TABLE II: PI controller parameters

\begin{tabular}{cc}
\hline Parameter & Value \\
\hline$\omega_{b d}$ & $1098.6 \mathrm{rad} / \mathrm{s}$ \\
$\omega_{b q}$ & $2197.2 \mathrm{rad} / \mathrm{s}$ \\
$k_{p d}$ & $\omega_{b d} L_{d}$ \\
$k_{i d}$ & $\omega_{b d} R$ \\
$k_{p q}$ & $\omega_{b q} L_{q}$ \\
$k_{i q}$ & $\omega_{b q} R$ \\
\hline
\end{tabular}

be obtained as

$$
i_{o q}^{2}=i_{o d}^{2}+\frac{\lambda_{p m}}{\left(L_{m d}-L_{m q}\right)} i_{o d} .
$$

Solving for $i_{\text {od }}$ gives

$$
i_{o d}=\frac{-\left(\lambda_{p m} \mp \sqrt{\lambda_{p m}^{2}+4 i_{o q}^{2}\left(L_{m d}-L_{m q}\right)^{2}}\right)}{2\left(L_{m d}-L_{m q}\right)} .
$$

Substituting this in the electromagnetic torque equation with a reference torque $\tau^{r e f}$ results in

$$
\left(L_{m d}-L_{m q}\right)^{2} i_{o q}^{4}+\frac{4 \tau \lambda_{p m}}{3 P} i_{o q}-\frac{16\left(\tau^{r e f}\right)^{2}}{9 P^{2}}=0 .
$$

Hence, for a given torque demand $\tau^{r e f}$, the solution within the machine constraints will be the reference q-axis current while the corresponding $\mathrm{d}$-axis current will be given by (29). As shown in the derivation above, since MTPA tries to maximize the electromagnetic torque per unit current, it also tries to minimize the current-dependent losses and hence the degradation, indirectly. The PI controllers in the MTPA control are tuned using loop shaping methods. The controller parameters $k_{p}$ and $k_{i}$ for the dq-current control loops can be obtained in terms of the bandwidth $\omega_{b}$ of the respective control loop as listed in Table II. More details on the characteristics of these two controllers can be found in [25] [45].

\section{B. Set up for torque transient and degradation analysis}

For a more realistic simulation a more accurate model, the higher-order model derived in the Appendix, is used as the plant in the control loop. In order to bring the machine to steady state, a torque reference step input of positive peak amplitude is initially given. Halfway through the simulation, another step input, that provides a negative maximum torque, is applied. This approach allows us to simulate the worst case scenario in the practical case, which represents the braking to standstill, while delivering the maximum torque. The results presented will be normalized to the peak torque.

The upper left and right entries of Fig. 7 show the torquespeed demands of the vehicle used in this study, during two well-known drive cycles: the New European Drive Cycle (NEDC) to represent urban driving and the Assessment and Reliability of Transport Emission Models and Inventory Systems (ARTEMIS) drive cycle to represent urban and motorway driving. According to their torque histograms (lower left and right figures respectively in Fig. 7), the torque demand in NEDC remains below $140 \mathrm{Nm}$, which is the maximum continuous torque output of the machine. However, in the ARTEMIS drive cycle, it reaches $210 \mathrm{Nm}$ frequently during
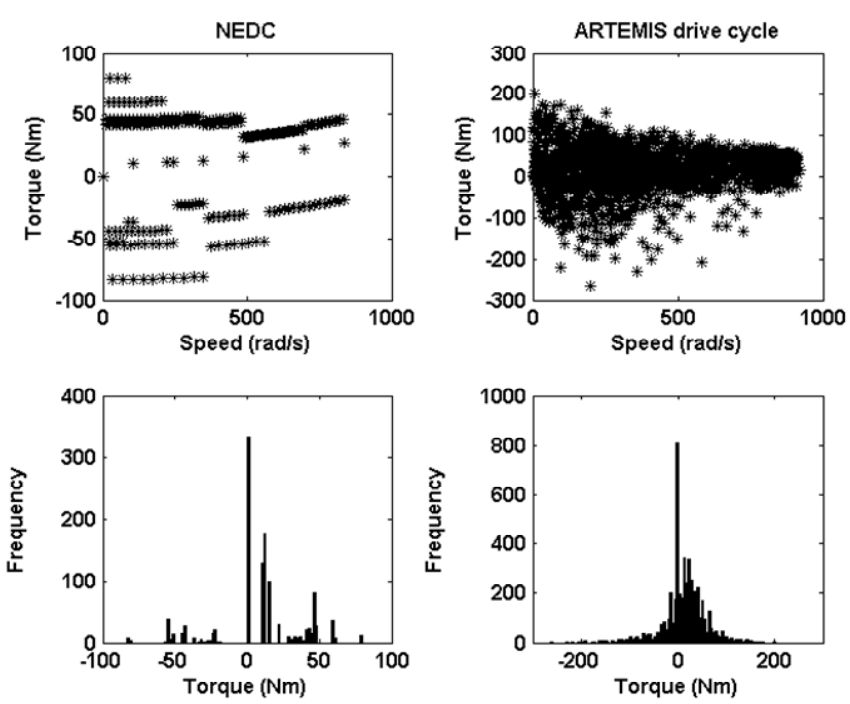

Fig. 7: Torque distribution during NEDC and ARTEMIS drive cycles.

braking and also $280 \mathrm{Nm}$ very rarely, which is the peak torque of the machine. In order to incorporate these torque demand conditions reasonably in the simulations, the procedure using the step inputs described above is repeated for a $50 \%$ (140 $\mathrm{Nm})$, a $75 \%(210 \mathrm{Nm})$ and a $100 \%(280 \mathrm{Nm})$ torque demand values.

\section{Torque transient results and analysis}

Both $i_{d}=0$ control and MTPA control have been used to compare the results with NMPC. However, beyond a $50 \%$ torque demand, $i_{d}=0$ control is unable to provide stable results and therefore it is not considered in the comparisons. Further, for the sake of brevity, resulting current, voltage and torque signals have been presented only for the $100 \%$ torque case, which is sufficient to observe the effectiveness of the NMPC during transients. Nevertheless, the degradation results have been presented for all the controllers in each simulation experiment. A value of $\alpha=0.5$ is used in the transient and drive cycle simulation studies.

In the $100 \%$ torque case shown in Fig.8, it is observed that at steady state, the torque producing current combinations (upper left and lower left in the figure) for the two controllers are different, hence showing the NMPC capabilities in exploiting the redundancy of the PMSM to its advantage. In addition, the $i_{o d}$ has a negative going peak for MTPA control and positive going peak for NMPC. Furthermore, for $i_{o q}$, the peak overshoot with MTPA control is $27.3 \%$ and for NMPC it is only $7.7 \%$. Due to the much lower overshoot in state transients, the torque transient with NMPC has only $3.5 \%$ overshoot, whereas it is $57.9 \%$ for MTPA control as shown in Fig. 9. In addition, it is observed that the NMPC is 3.4 times faster to settle. As expected, the optimal solution obtained by NMPC results in faster operation with much lower overshoot against the PI counterpart in the MTPA control. This will have a positive effect on reducing degradation. The voltages shown in the upper two figures of Fig. 10, $v_{d}$ has $36.4 \%$ overshoot with MTPA control, whereas with NMPC, the overshoot is 

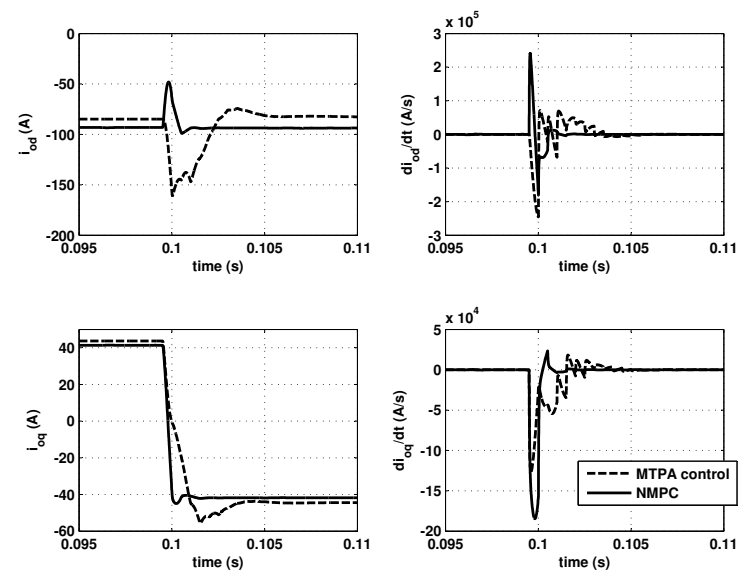

Fig. 8: Step response comparison of states at maximum to minimum peak torque transient

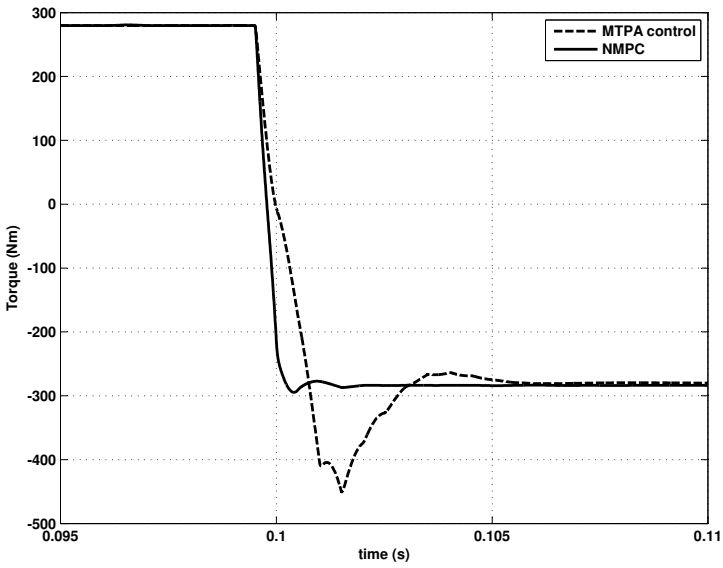

Fig. 9: Comparison of torque response during maximum to minimum peak transient

only $2.2 \%$. In addition, both $v_{d}$ and $v_{q}$ hit the voltage limit with the MTPA control, whereas it stays within limits with the NMPC. As an additional advantage, the lower voltage enables a smaller DC link capacitor in the converter for NMPC. The corresponding PMSM currents shown in the lower two figures in Fig. 10 reveal that the maximum $i_{d}$ is lower in NMPC (73.4 A). In addition, $i_{q}$ is 3.4 times faster in NMPC, which will contribute to significantly lower degradation, while requiring lower current capability from the energy storage system.

It is also noted that re-tuning of the linear PI controllers of the MTPA control does not help. Rise time can be made faster only at the expenses of increased overshoot. Furthermore, only the NMPC scheme is able to satisfy the nonlinear constraints a priori.

\section{Degradation results and analysis}

Degradation associated with each of the above cases is presented in Fig. 11. For the $50 \%$ torque case, due to the large overshoot and high q-current demand, the degradation of $i_{d}=0$ control is $356 \%$ higher than that of MTPA control and NMPC. The degradations in NMPC and MTPA controllers
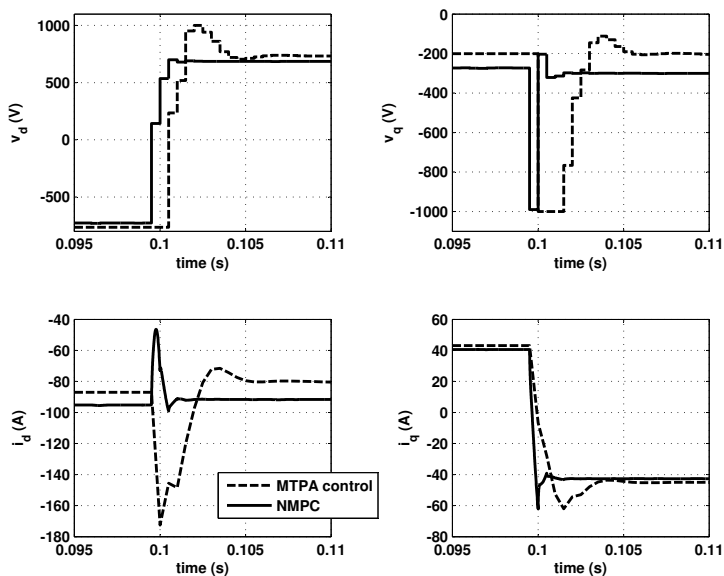

Fig. 10: Machine voltage and current comparisons at maximum to minimum peak torque transient.

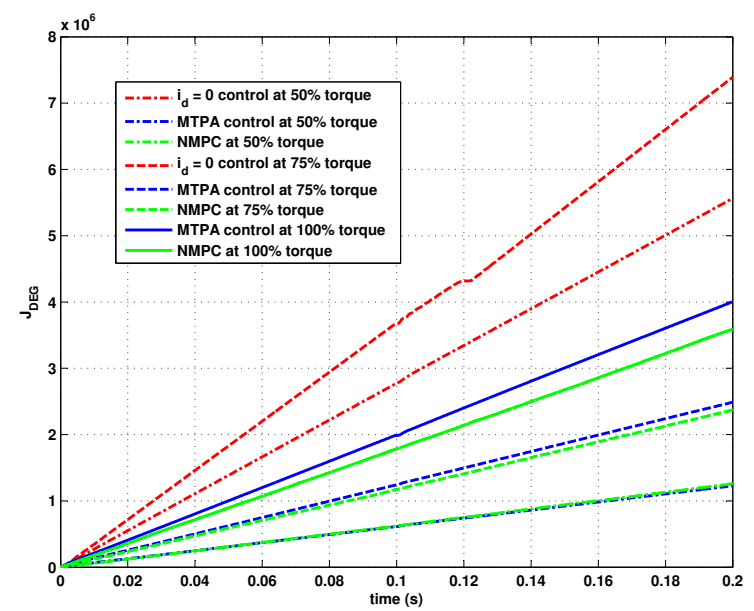

Fig. 11: Degradation results comparison

are almost the same in this case. For the $75 \%$ torque case, due to faster state and torque transients, but lower overshoots, the degradation is $4.6 \%$ lower in NMPC as compared to MTPA control. In the $100 \%$ torque case also, faster state and input transients with lower overshoots contribute significantly to keep the degradation to a minimum for NMPC, where it is $11.1 \%$ lower than the MTPA control.

It is noted that until the torque demands are in the range of $50 \%$ of the peak torque, the NMPC and MTPA control give similar degradation results. The gain in reducing degradation is significant if the machine torque demand is above $50 \%$ of the peak torque very often.

\section{E. Analysis of the impact of $\alpha$}

One of the main advantages of having a multi-objective cost function is the flexibility in choosing the weighting factor $\alpha$. Fig. 12 shows how the degradation varies for the same transient study in the $100 \%$ torque case, when $\alpha$ is varied from 0.1 to 0.9 in steps of 0.1 . It is clearly seen that in all cases the degradation with NMPC is lower than the MTPA control. The main reason being that the NMPC models and penalizes the degradation in the cost function, while the MTPA does not. 


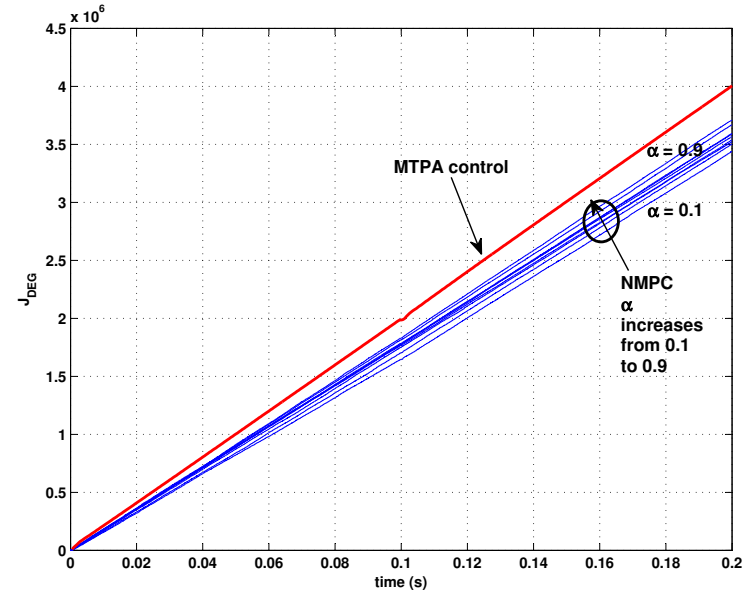

Fig. 12: Degradation results comparison with MTPA control when $\alpha$ is changed in NMPC

TABLE III: Vehicle parameters [46]

\begin{tabular}{cc}
\hline Parameter & Value \\
\hline Mass $M$ & $1521 \mathrm{~kg}$ \\
Drag coefficient $C_{d}$ & 0.29 \\
Area facing wind $A$ & $2.27 \mathrm{~m}^{2}$ \\
Gravitational acceleration $g$ & $9.81 \mathrm{~ms}^{-2}$ \\
Tire radius $r$ & $0.316 \mathrm{~m}$ \\
Gear ratio $G R$ & 7.94 \\
\hline
\end{tabular}

\section{DRIVE CYCLE PERFORMANCE ANALYSIS}

In this section, the performance of the NMPC scheme is compared with the MTPA control using the NEDC and ARTEMIS drive cycles as torque demands. The vehicle considered in this case study has parameters equivalent to a commercially available Nissan ${ }^{\mathrm{TM}}$ Leaf electric car.

\section{A. Torque demand calculation}

The torque demand of the electric vehicle during the drive cycle is calculated using vehicle dynamics. A simplified vehicle model is used where it is assumed that the vehicle travels on a flat road surface with uniform friction. The combination of forces acting on the vehicle consist of the traction force from the PMSM $F_{t}$, air resistance force on the body of the car $F_{a i r}$ and rolling resistance from the road surface $F_{r r}$. The mass of the vehicle is $M$ and $v$ is the instantaneous velocity. Applying Newton's second law of motion gives

$$
F_{t}-F_{a i r}-F_{r r}=M \frac{d v}{d t} \text {. }
$$

The air resistance force is given by $F_{a i r}=\frac{1}{2} \rho C_{d} A v^{2}$, and the rolling resistance is given by $F_{r r}=M g \mu$, where the definitions of the symbols and the numerical values of each of the parameters used in the above equations are given in TABLE III. Assuming a loss free gear box, the torque demand, which is the reference torque $\tau^{r e f}$ on the machine shaft can be obtained as $\tau^{r e f}=\frac{F_{t} r}{G R}$. The resulting torque demands are shown (in red) in Fig. 13 together with the respective speeds (in black) for NEDC (upper) and ARTEMIS drive cycle

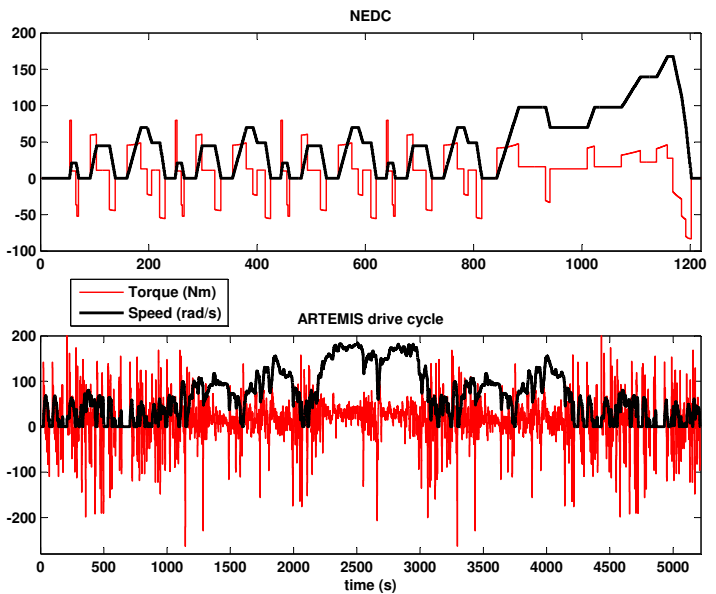

Fig. 13: Torque demand for the drive cycles.
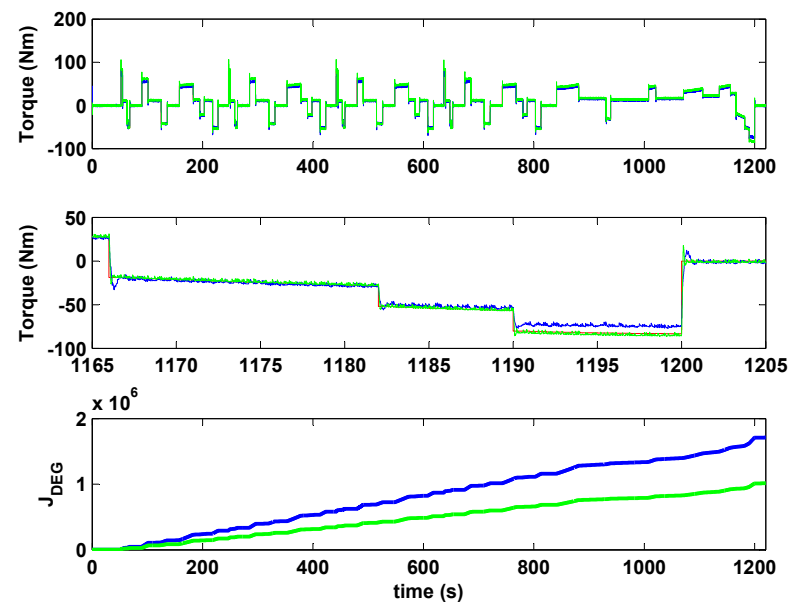

Fig. 14: Degradation comparison for NEDC. Blue: MTPA control, Green: NMPC

(lower) respectively. (The NEDC speed has been divided by 5 to improved visibility.)

\section{B. Degradation comparison}

By applying the torque demands calculated above on the PMSM, the performance of the NMPC and MTPA control is compared.

1) NEDC: The torque output of the two controllers for the full NEDC is shown in the upper plot of Fig. 14. Both the controllers let the machine deliver the torque according to the requirements of the NEDC. There are differences, which lie in particular during the transients. The section from time $=1192 \mathrm{~s}$ to time $=1202 \mathrm{~s}$ is zoomed in and shown in the middle plot of Fig. 14. There, it is observed that both MTPA control and NMPC follow the reference torque (red) when the torque demand is lower. However, when it is higher (reaching $\pm 50 \mathrm{Nm}$ ), the torque magnitude produced by MTPA control is lower. The reason for this can be observed in Fig.15, where $i_{d}$ (upper), $i_{q}$ (middle) and $i=\sqrt{i_{d}^{2}+i_{q}^{2}}$ (lower) are shown. It is very clear that the lower torque is due to the lower torque 
producing current $i_{q}$ with MTPA control, when the torque demand is higher between time $=1190 \mathrm{~s}$ and time $=1200$ s. The current $i$, which is the cause of losses, is higher with MTPA control than with NMPC. The current $i_{q}$ rise time is larger with MTPA control during the transients shown in Fig. 15 (middle) at time $=1200 \mathrm{~s}$. This also explains why the degradation with NMPC is lower. Hence, NPMC exploits the over actuated nature of the PMSM to mitigate the degradation, while tracking the torque demand as standards controllers do.
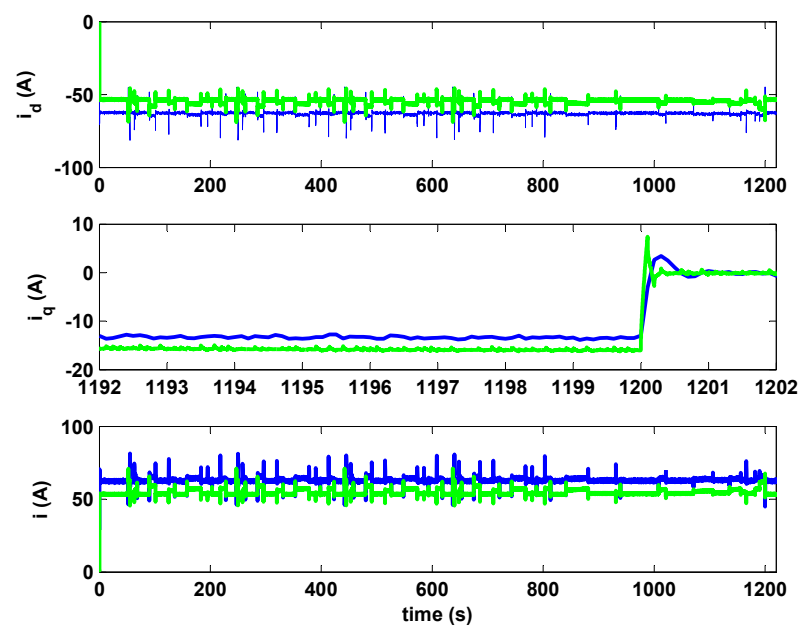

Fig. 15: PMSM currents during NEDC, Blue: MTPA control, Green: NMPC

2) ARTEMIS: The same procedure as in NEDC is repeated here. The upper plot of Fig. 16 shows the torque responses of the two controllers for the full ARTEMIS drive cycle. Both controllers are able to track the desired torque profile but both fail to reach the peak torque demands (red). The middle plot of the Fig. 16 shows the zoomed in version from time $=1100 \mathrm{~s}$ to time $=1105 \mathrm{~s}$, where it is very clearly seen that the NMPC is faster with same or lower overshoot than the MTPA control, which is the main reason for lower degradation as observed in the lower plot of Fig. 16.

Unlike in the NEDC, the steady state performance of $i_{q}$ from both the controllers is similar as shown in Fig. 17 (middle). Consequently, the torque-tracking performance is also similar. However, similar to the NEDC case, $i$ as shown in Fig.17 (lower) remains lower, hence the losses are lower, explaining why the degradation is also lower with NMPC in this case.

\section{Improvements in remaining useful life}

Assuming a design life time of 15 years (i.e. $t_{\text {life }}=15$ years) of operational time for the PMSM of rated power $P_{\text {rated }}=80 \mathrm{~kW}$ with a rated efficiency $\eta=90 \%$, the CLRs are calculated when the vehicle is running on the two drive cycles. The durations of the drive cycles are $1220 \mathrm{~s}$ and 5218 $\mathrm{s}$ respectively. TABLE IV shows the RUL by using the NMPC and the MTPA control assuming that the vehicle run on the same drive cycle throughout $t_{\text {life }}$.
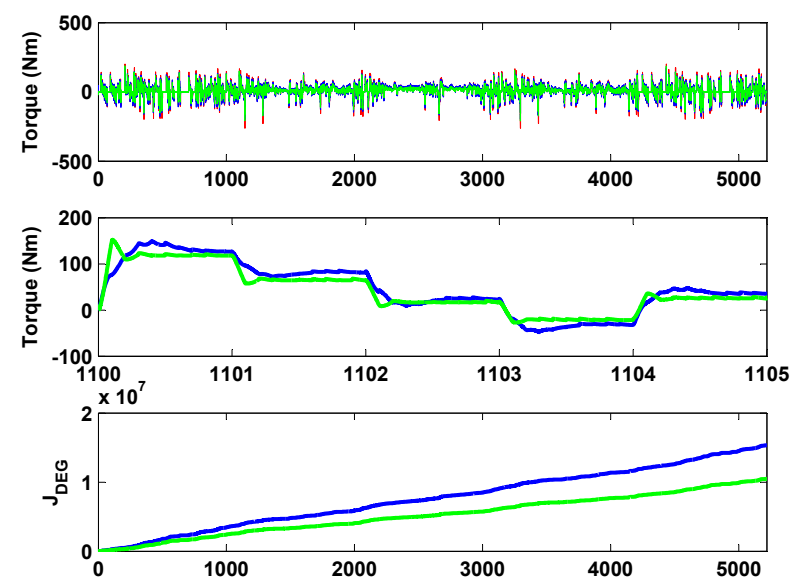

Fig. 16: Degradation comparison for ARTEMIS drive cycle. Red: $\tau^{r e f}$, Blue: MTPA control, Green: NMPC
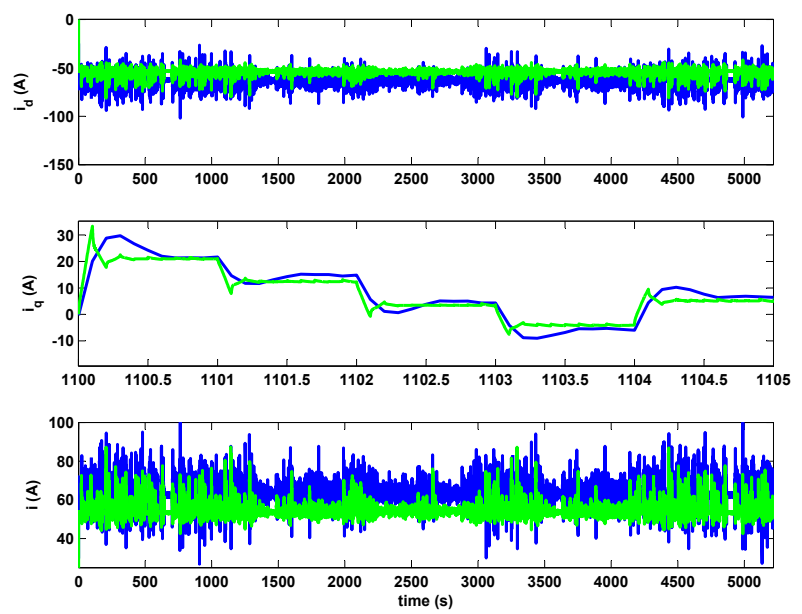

Fig. 17: PMSM currents during ARTEMIS. Red: $\tau^{r e f}$, Blue: MTPA control, Green: NMPC

TABLE IV: Remaining Useful Life (RUL)

\begin{tabular}{cc}
\hline Parameter & Value \\
\hline NMPC on NEDC & 0.9533 \\
MTPA control on NEDC & 0.9211 \\
NMPC ARTEMIS & 0.8870 \\
MTPA control on ARTEMIS & 0.8346 \\
\hline
\end{tabular}

Accordingly, for NEDC, the RUL is higher by $7.6 \%$ (i.e. almost one year of operational time) when using NMPC over MTPA control. Furthermore, for the ARTEMIS drive cycle, the RUL is higher by $15.7 \%$ (i.e. more than 1.5 years of operational time) when using NMPC over MTPA control.

\section{CONCLUSIONS AND FUTURE WORK}

By exploiting the inherent redundancy in actuation in electric machines, we have shown how their degradation can be minimized, while closed loop tracking performance maintained, via constrained optimal feedback control. A multiobjective optimal control problem has been formulated and 


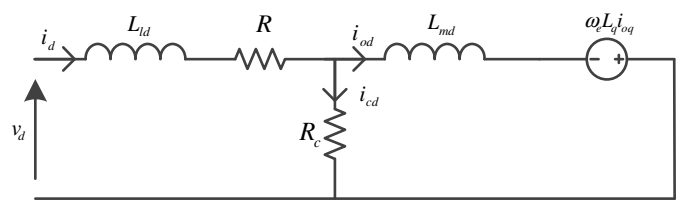

(a)

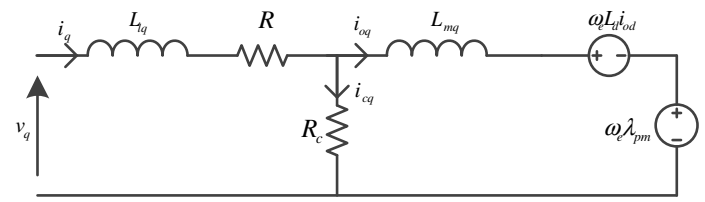

(b)

Fig. 18: Equivalent circuit of the higher-order machine model.

implemented via an NMPC scheme. A tuning parameter in the cost function allows to trade off degradation and closed loop performance. It was observed, in simulation experiments using a higher-order model for the plant, that NMPC outperforms the de-facto MTPA controller because of its ability of providing an optimal solution rather than a heuristic one. Algorithm optimization for real-word applications could be the subject of future research.

\section{APPENDIX: HIGHER-ORDER MACHINE MODEL}

Here we present a novel higher-order electric machine model that is used in the simulation experiments to have a better representation of the plant. In this model we separate the magnetizing inductance from the leakage inductance and insert the core resistance in between. This relaxes the simplifications made in combining those inductances in deriving the conventional lumped parameter PMSM model [30] presented before, resulting in a closer representation of the real machine. In addition, the iron losses during transients are represented more accurately.

The magnetizing inductance $L_{m}$ and leakage inductance $L_{l}$ are separated and the core loss resistance $R_{c}$ is included in between them to form a ' $\mathrm{T}$ ' network. Accordingly, the d-axis inductance consists of two components, namely the leakage inductance $L_{l d}$ and the magnetizing inductance $L_{m d}$ where the equivalent circuit is shown in Fig. 18 (a). Similarly, the q-axis inductance also consists of the corresponding two components, i.e. the leakage inductance $L_{l q}$ and the magnetizing inductance $L_{m q}$, whose equivalent circuit is shown in Fig. 18 (b). The stator electrical frequency is given by $\omega_{e}$ and $\lambda_{p m}$ is the flux linkage of the permanent magnets of the machine. The application of Kirchhoff's voltage and current laws in the daxis circuit shown in Fig. 18 (a) results in

$$
\begin{aligned}
v_{d} & =L_{l d} \frac{d i_{d}}{d t}+R i_{d}+L_{m d} \frac{d i_{o d}}{d t}-\omega_{e} L_{q} i_{o q} \\
i_{d} & =i_{o d}+i_{c d} \\
i_{c d} & =\frac{L_{m d} \frac{d i_{o d}}{d t}-\omega_{e} L_{q} i_{o q}}{R_{c}} .
\end{aligned}
$$

Following the same procedure as in the d-axis circuit, by applying the Kirchhoff's voltage and current laws in the qaxis circuit shown in Fig. 18 (b) results in

$$
\begin{aligned}
v_{q} & =L_{l q} \frac{d i_{q}}{d t}+R i_{q}+L_{m q} \frac{d i_{o q}}{d t}+\omega_{e} L_{d} i_{o d} \\
& +\omega_{e} \lambda_{p m} \\
i_{q} & =i_{o q}+i_{c q} \\
i_{c q} & =\frac{L_{m q} \frac{d i_{o q}}{d t}+\omega_{e} L_{d} i_{o d}+\omega_{e} \lambda_{p m}}{R_{c}} .
\end{aligned}
$$

By defining the input $u$, state $x$ and output $y$ vectors as

$$
\begin{aligned}
u & =\left[v_{d}, v_{q}\right]^{T} \\
x & =\left[i_{o d}, \dot{i}_{o d}, i_{o q}, \dot{i}_{o q}\right]^{T} \\
y & =\left[i_{d}, i_{q}\right]^{T}
\end{aligned}
$$

the system can be expressed in state space form as

$$
\begin{aligned}
\dot{x} & =f(x, u) \\
y & =h(x, u),
\end{aligned}
$$

where $f=\left[f_{1}, f_{2}, f_{3}, f_{4}\right]^{T}$ is

$$
\begin{aligned}
f_{1} & =x_{2} \\
f_{2} & =-\left(\frac{R R_{c}}{L_{l d} L_{m d}}\right) x_{1} \\
& -\frac{R_{c}}{L_{l d} L_{m d}}\left(L_{l d}+\left(1+\frac{R}{R_{c}}\right) L_{m d}\right) x_{2} \\
& +\frac{R_{c}}{L_{l d} L_{m d}} \omega_{e} L_{q}\left(1+\frac{R}{R_{c}}\right) x_{3} \\
& +\omega_{e} \frac{L_{q}}{L_{m d}} x_{4}+\frac{R_{c}}{L_{l d} L_{m d}} u_{1} \\
f_{3} & =x_{4} \\
f_{4} & -\frac{R_{c}}{L_{l q} L_{m q}} \omega_{e} L_{d}\left(1+\frac{R}{R_{c}}\right) x_{1}-\omega_{e} \frac{L_{d}}{L_{m q}} x_{2} \\
- & \left(\frac{R R_{c}}{L_{l q} L_{m q}}\right) x_{3} \\
- & \frac{R_{c}}{L_{l q} L_{m q}}\left(L_{l q}+\left(1+\frac{R}{R_{c}}\right) L_{m q}\right) x_{4} \\
+ & \frac{R_{c}}{L_{l q} L_{m q}} u_{2}-\frac{R_{c}}{L_{l d} L_{m d}} \omega_{e} \lambda_{p m}\left(1+\frac{R}{R_{c}}\right)
\end{aligned}
$$

and $h=\left[h_{1}, h_{2}\right]^{T}$ is

$$
\begin{aligned}
h_{1} & =x_{1}+\frac{L_{m d}}{R_{c}} x_{2}-\omega_{e} \frac{L_{q}}{R_{c}} x_{3} \\
h_{2} & =\omega_{e} \frac{L_{d}}{R_{c}} x_{1}+x_{3}+\frac{L_{m q}}{R_{c}} x_{4}+\omega_{e} \frac{\lambda_{p m}}{R_{c}} .
\end{aligned}
$$

\section{ACKNOWLEDGMENT}

Authors acknowledge the financial support from EPSRC through the Developing FUTURE Vehicles grant $(\mathrm{EP} / \mathrm{I} 038586 / 1)$ and Impact Acceleration Account grant (EP/K503927/1).

\section{REFERENCES}

[1] K.T. Chau, C.C. Chan, C. Liu, Overview of Permanent-Magnet Brushless Drives for Electric and Hybrid Electric Vehicles, IEEE Trans. Ind. Electron., vol. 55, no. 6, pp.2246 - 2257, 2008. 
[2] K. Kamiev, J. Montonen, M.P. Ragavendra, J. Pyrhonen, J.A. Tapia, M. Niemela, Design Principles of Permanent Magnet Synchronous Machines for Parallel Hybrid or Traction Applications, IEEE Trans. Ind. Electron., vol. 60 , no. 11 , pp. 4881 - 4890, 2013.

[3] K. I. Laskaris and A. G. Kladas, Internal Permanent Magnet Motor Design for Electric Vehicle Drive, IEEE Trans. Ind. Electron., vol. 57, no. 1, pp. $138-145,2010$

[4] N. Mutoh, Driving and Braking Torque Distribution Methods for Frontand Rear-Wheel-Independent Drive-Type Electric Vehicles on Roads With Low Friction Coefficient,IEEE Trans. Ind. Electron., vol. 59, no. 10, pp. 3919 - 3933, 2012.

[5] J. Nerg, M. Rilla, V. Ruuskanen, J. Pyrhonen, and S. Ruotsalainen, DirectDriven Interior Magnet Permanent-Magnet Synchronous Motors for a Full Electric Sports Car, IEEE Trans. Ind. Electron., vol. 61, no. 8, pp. 4286 - 4294, 2014

[6] S.I. Kim, J. Cho, S. Park, T. Park, S. Lim, Characteristics comparison of a conventional and modified spoke-type ferrite magnet motor for traction drives of low-speed electric vehicles, IEEETrans. Ind. Electron., vol. 49, no. 6, pp. 2516-2523, 2013.

[7] F. Zhu, L. Chen, C. Yin, Design and analysis of a novel multimode transmission for a HEV using a single electric machine, IEEE Trans. Veh. Techno., vol. 62, no. 3, pp. 1097 - 1110, 2013.

[8] P. Werynski, D. Roger, R. Corton, J. F. Brudny, Proposition of a new method for in-service monitoring of the aging of stator winding insulation in AC motors, IEEE Trans. Energy Conv., vol. 21, no. 3, pp. 673 - 681, 2006.

[9] N. Lahoud, J. Faucher, D. Malec, P. MAUSSION, Electrical Aging of the Insulation of Low-Voltage Machines: Model Definition and Test With the Design of Experiments, IEEE Trans. Ind. Electron., vol. 60, no. 9, pp. $4147-4155,2013$

[10] O. Winter, C. Kral, E. Schmidt, Augmented Temperature Degrading Effect of Rare Earth Magnets Arranged in Segmented Halbach Arrays, IEEE Trans. Magnetics, vol. 48, no. 11, pp. 3335 - 3338, 2012

[11] J.D. McFarland, T.M. Jahns, Influence of d- and q-axis currents on demagnetization in PM synchronous machines, Proc. 2013 IEEE Energy Conversion Congress and Exposition, Sept. 2013.

[12] S. Xikai, C. Ming, Thermal Analysis and Cooling System Design of Dual Mechanical Port Machine for Wind Power Application, IEEE Trans. Ind. Electron., vol. 60, no. 5, pp. 1724 - 1733, 2013.

[13] A. Soualhi, K. Medjaher, N. Zerhouni, Bearing Health Monitoring Based on HilbertHuang Transform, Support Vector Machine, and Regression, IEEE Trans. Inst. and Measurement, vol. 64, no. 1, pp.52 - 62, 2015.

[14] F. Rufus, S. Lee, A. Thakker, S. Field, Advanced Diagnostics of Aircraft Electrical Generators, SAE Int. J. Aerosp. vol. 1, no.1, pp 1064 - 1070, 2009.

[15] R. Findeisen, F. Allgöwer, L. T. Biegler, Assessment and future directions of nonlinear model predictive control - 2007 - Springer

[16] M. Böck and A. Kugi, Real-time Nonlinear Model Predictive PathFollowing Control of a Laboratory Tower Crane, IEEE Transactions on Control Systems Technology, vol. 22, no. 4, pp. 1461-1473, July 2014.

[17] E. Kayacan, E. Kayacan, H. Ramon and W. Saeys, Learning in Centralized Nonlinear Model Predictive Control: Application to an Autonomous Tractor-Trailer System, IEEE Transactions on Control Systems Technology, vol. 23, no. 1, pp. 197-205, Jan. 2015.

[18] J. Lemmens, P. Vanassche, J. Driesen, PMSM Drive Current and Voltage Limiting as a Constraint Optimal Control Problem, IEEE Jrnl. Emerg. Sel. Topics in Power Electron., vol. PP, no.99, pp.1-13, 2014.

[19] P. Cortes, M. P. Kazmierkowski, R. M. Kennel, D. E. Quevedo and J. Rodriguez, Predictive Control in Power Electronics and Drives, in IEEE Transactions on Industrial Electronics, vol. 55, no. 12, pp. 4312-4324, Dec. 2008.

[20] J. Rodriguez et al., State of the Art of Finite Control Set Model Predictive Control in Power Electronics, in IEEE Transactions on Industrial Informatics, vol. 9, no. 2, pp. 1003-1016, May 2013.

[21] M. Preindl and S. Bolognani, Model Predictive Direct Speed Control with Finite Control Set of PMSM Drive Systems, in IEEE Transactions on Power Electronics, vol. 28, no. 2, pp. 1007-1015, Feb. 2013.

[22] R. P. Aguilera and D. E. Quevedo, On stability and performance of finite control set MPC for power converters, Predictive Control of Electrical Drives and Power Electronics (PRECEDE), 2011 Workshop on, Munich, 2011, pp. 55-62.

[23] Seung-Ki Sul, Control of Electric Machine Drive Systems, Wiley 2011

[24] J. Chiasson, Modeling and High Performance Control of Electric Machines John Wiley \& Sons, 2005

[25] B. K. Bose, Ed., Power Electronics and Variable Frequency Drives. Piscataway, NJ: IEEE Press, 1996.

[26] C. Cavallaro, A.O. Di Tommaso, R. Miceli, A, Raciti, G.R. Galluzzo, and M. Trapanese, Efficiency Enhancement of Permanent-Magnet Syn- chronous Motor Drives by Online Loss Minimization Approaches, IEEE Trans. Ind. Electron., vol. 52, no. 4, pp. 1153 - 1160, 2005

[27] J. Lee, K. Nam, S. Choi, S. Kwon, Loss-minimizing control of PMSM with the use of polynomial approximations, IEEE Trans. Power Electron., vol 24, no 4, 2009, pp. 1071-1082

[28] M.N. Uddin, R.S. Rebeiro, Online efficiency optimization of a fuzzylogic-controller-based IPMSM drive, IEEE Trans. Ind. Electron., vol. 47, no. 2, pp. 1043 - 1050, 2011

[29] C. T. Pan, S. M. Sue, A linear maximum torque per ampere control for IPMSM drives over full-speed range, IEEE Trans. Energy Conversion, vol. 20, no. 2, pp. 359 - 366, 2005.

[30] L. Samaranayake, Y. K. Chin, Speed Synchronized Control of Permanent Magnet Synchronous Machine Drive Systems with Field Weakening, Proc. IASTED Intl. Conf. Power and Energy Systems, Marbella, Spain, September 2003.

[31] L. Samaranayake, S. Alahakoon, K. S. Walgama, Speed Controller Strategies for Distributed Motion Control via Ethernet, Proc. 18th IEEE Intl. Symp. Intelligent Control, Texas Houston, USA, October 2003.

[32] L. Samaranayake, Y. K. Chin, S. Alahakoon, Distributed Control of Permanent Magnet Synchronous Motor Drive Systems, Proc. IEEE Power Electronics and Drive Systems Conf., Singapore, November 2003.

[33] L. Samaranayake, S. Longo, Cost Functions for Degradation Control of Electric Motors in Electric Vehicles, Proc. in European Control Conference (ECC), June 2015.

[34] L. Samaranayake, S. Longo, Nonlinear Model Predictive Control for Traction Motor Degradation Minimization, Proc. in $54^{\text {th }}$ IEEE Conference on Decision Control (CDC) Dec. 2015.

[35] J. B. Rawlings, D. Q. Mayne, Model Predictive Control: Theory and Design, Nob Hill Publishing, 2009

[36] H. Borhan, A. Vahidi, A. M. Phillips, M. L. Kuang, I. V. Kolmanovsky and S. Di Cairano, MPC-Based Energy Management of a Power-Split Hybrid Electric Vehicle, IEEE Transactions on Control Systems Technology, vol. 20, no. 3, pp. 593-603, May 2012.

[37] H. Guzman, M. J. Duran, F. Barrero, B. Bogado, S. Toral, Speed Control of Five-Phase Induction Motors With Integrated Open-Phase Fault Operation Using Model-Based Predictive Current Control Techniques, IEEE Trans. Ind. Electron., vol. 61, no. 9, pp.4474 - 4484, 2014.

[38] S.J. Moura, H.K. Fathy, D.S. Callaway, J.L. Stein, A Stochastic Optimal Control Approach for Power Management in Plug-In Hybrid Electric Vehicles, IEEE Trans. Control Systems, vol. 19, no. 3, pp. 545 - 555, 2011.

[39] S.J. Moura, D.S. Callaway, H.K. Fathy, J.L. Stein, Tradeoffs between battery energy capacity and stochastic optimal power management in plug-in hybrid electric vehicles, Journal of Power Sources, vol. 195, no 9, pp 2979 - 2988, 2010.

[40] M. Sumislawska, K. N. Gyftakis, D. F. Kavanagh, M. McCulloch, K. J. Burnham and D. A. Howey, The impact of thermal degradation on electrical machine winding insulation, 2015 IEEE 10th International Symposium on Diagnostics for Electrical Machines, Power Electronics and Drives pp. 232-238.

[41] I. Semsei, On the nature of aging, Mechanisms of Ageing and Development, vol 117, Issues 13, Aug. 2000, pp $93-108$

[42] ICLOCS official web site http: //www.ee.ic.ac.uk/ICLOCS/ [Accessed 22 November 2014]

[43] ICLOCS projects web site https : //projects.coin or.org/IpoptPleaseCiteUs [Accessed 22 November 2014]

[44] P. Falugi, E. Kerrigan, E. V. Wyk. Imperial College London Optimal Control Software User Guide (ICLOCS), Department of Electrical and Electronic Engineering, Imperial College London, UK, May 2010.

[45] Mohan, N., Electric Drives, An Integrative Approach, 3rd Edition 2003.

[46] Nissan Motor Corporation website, www.nissanusa.com [Accessed 10 April 2015]

[47] Suardi, A., Longo, S., Kerrigan, E. C., \& Constantinides, G. A. (2013). Energy-aware MPC co-design for DC-DC converters. In European Control Conference (ECC13), 2013. Zurich, Switzerland

[48] Suardi, A., Longo, S., Kerrigann, E. C., \& Constantinides, G. A. (2016). Explicit MPC: Hard constraint satisfaction under low precision arithmetic. Control Engineering Practice, 47, 6069.

[49] FORCESPro web site https : //www.embotech.com/FORCES Pro [Accessed 27 August 2016] 
2017-01-17

\section{Degradation control for electric vehicle machines using nonlinear model predictive control}

Samaranayake, Lilantha

IEEE

Samaranayake L, Longo S, Degradation control for electric vehicle machines using nonlinear model predictive control, IEEE Transactions on Control Systems Technology, Vol. 26, Issue 1, January 2018, pp. 89-101

http://dx.doi.org/10.1109/TCST.2016.2646322

Downloaded from Cranfield Library Services E-Repository 